\title{
A HUGE CEMENTO-OSSIFYING FIBROMA OF PARANASAL SINUS: A CASE REPORT
}

\author{
Ibrahim Erdim ${ }^{1}$, Zahide Mine Yazici', Rasim Yilmazer', Nurten Sever ${ }^{2}$, Fatma Tulin Kayhan ${ }^{1}$
}

Bakırköy Dr. Sadi Konuk Training and Research Hospital, Istanbul, Turkey: Department of Otorhinolaryngology ${ }^{1}$; Department of Pathology2

Summary: Cemento-ossifying fibroma is a well-bordered, slow-growing, benign fibro-osseous disease. Although its localization is generally in the mandible, it can be seen in any area of the craniofacial region. Radiology and histopathology help to diagnose the condition. Treatment is based on close observation and/or surgical excision. In this case, we report the case of a 62 -year-old male patient who had a large radiological appearance, cemento-ossifying fibroma in the paranasal sinuses.

Key words: Benign fibro-osseous disease; Cemento-ossifying fibroma

\section{Introduction}

Fibro-osseous lesions of the craniofacial region consist of varying amounts of fibrous stroma or osseous tissue. They involve three kinds of diseases called fibrous dysplasia, ossifying fibroma and osteoma $(1,2)$.

Cemento-ossifying fibroma (COF), a synonym of ossifying fibroma, is a well-bounded benign fibro-osseous disease (BFOD) as clarified by the World Health Organization (WHO) in 2005 (3). Although its localization is generally mandible (75-89\%) and maxilla, it can be seen in any other craniofacial region like paranasal sinuses, nasal cavity, temporal bone and occipital bone (4). Symptoms usually depend on localization and size of the fibroma but it may be asymptomatic. Nasal obstruction, proptosis, chronic rhinosinusitis, facial deformity, brain expansion, cerebellar constriction and visual disorder caused by compression of the optic nerve are the symptoms of COF $(2,5)$.

Frequently it is noticed on dental examination, radiographs or CT images incidentally as an expanding mass. COF is said to grow slowly and some authors have declared its growth speed to be nearly $0.5 \mathrm{~mm}$ in a year (6). The average age at diagnosis of COF is third and fourth decades and a female predilection is viewed (female-to-male ratio of $1.6: 1)(7)$. We report a case of COF in a rare localization and huge mass.

\section{Case Presentation}

A 62-year-old man presented with right-sided nasal obstruction for many years. No other complaint was
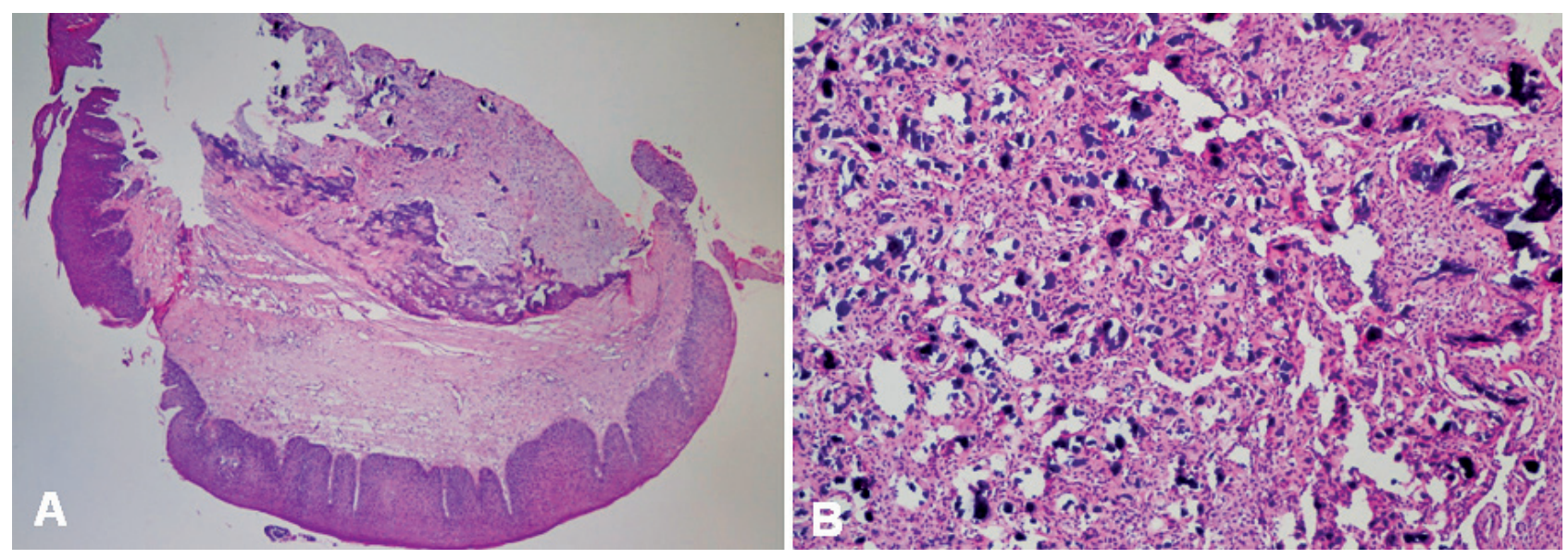

Fig. 1: Fibrocellular lesion under nonkeratinized squamous epithelium (hematoxylin-eosin, 40X) (A). Basophilic globules of calcified lesions called cementum within cellular fibrous connective tissue stroma (hematoxylin-eosin, 100X) (B). 
determined. Upon inspection of facial structure, his right eye was proptosed and displaced inferolaterally. Visual acuity, fundus examination, pupillary responses and extra ocular movements were normal. Cranial nerve examination and other neurological findings were normal.

Physical examination revealed a stiff mass in right nasal cavity which was almost totally obstructed. He had no medical history of malignancy, chronic disease, operation or trauma. His laboratory tests were normal.

In macroscopic biopsy material punched in the right nasal cavity, broken white-coloured tissue specimen was seen. Microscopic examination of the excised tissue revealed a nodular dense lesion placed under hyperplastic squamous epithelium. The lesion contains fibroblastic stroma, acellular osteoid material, and focal round to ovoid basophilic calcifications called 'cementum' and bone spicules (figure 1).

Computed tomography showed a lesion extending from right nasal cavity to the choana, laterally made right orbital medial wall protrusion, superiorly reached brain parenchyma and medially pushed nasal septum to the left. Its size

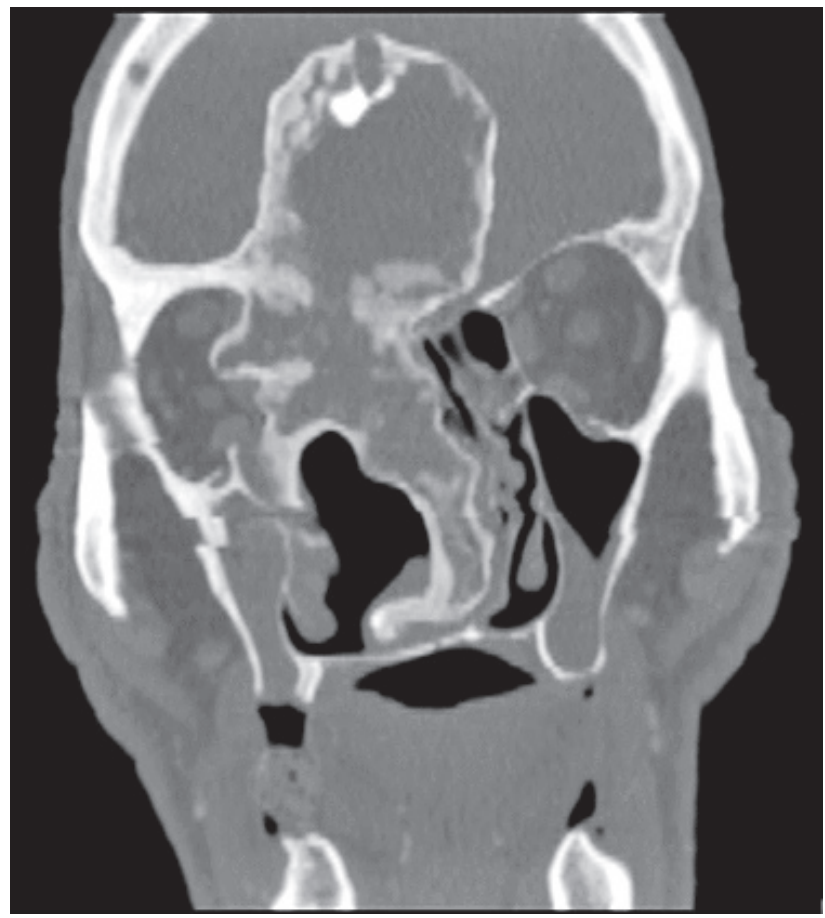

Fig. 2: Coronal reformatted CT scan image of the face in the bone algorithm demonstrate a lobulated, well circumscribed, peripherally calcified mass centered in the right parasagittal anterior cranial fossa floor. Superiorly it extends along the interhemispheric fissure and inferiorly involves and causes expansion of superior and middle right nasal turbinates, obliterating the ethmoid air cells. Laterally it involves the medial wall of the right orbit, causing lateral bowing leading and mass effect on the intraorbital contents. was $114 \times 55 \times 42$ millimeter (superoinferior $\mathrm{x}$ anteroposterior $\mathrm{x}$ mediolateral). The lesion had some calcification foci in its periphery and central zone. It didn't destruct adjacent bones and its character was expansible (figure 2).

Magnetic resonance imaging showed a mass filled up right maxillary sinus, nasal cavity and ethmoid cellules. The medial wall of right maxillary sinus wasn't seen. The lesion pushed right frontal brain lobe to the left side, entered into orbita from medial wall so displaced bulbus oculi and optic nerve laterally. The mass showed hypo intense signal on $\mathrm{T} 1$ and hyper intense signal on T2-weighted images. With contrast agent intracranial part of lesion (especially surrounding component) got signal intensity (figure 3).

The patient was informed about operation's possible complications in areas such as orbita, skull base, optic nerve and frontal brain lobe. He had no significant complaint and he refused to be operated on. During a two-year follow up, no additional symptom or complication was detected.

\section{Discussion}

COF is a well-demarcated lesion composed of fibrocellular tissue and mineralized material (3).

The certain etiopathogenesis of COF is unknown but two theories are claimed for this disease. First one is irritants such as trauma, micro-organisms, plaque, calculus, dental restorations and dental appliances are thought to affect periodontal membrane (8). It is acceptable for most cases that are located in mandible and maxilla. But it confuses the fact of how COF is seen in paranasal sinuses, nasal cavity, temporal bone and other localizations where there aren't any cementum producing cells. So it is reasonable to believe the second theory that ectopic periodontal membrane or primitive mesenchymal cells enlarge, then produce fibro-osseous tissue and cementum (9).

Pathologic examination is one of the diagnosis methods. COF develops centrifugally and gets a round or oval shape. Macroscopically, COF displays a whitish-grayish solid mass. The microscopic examination of the COF shows a dense, cellular, fibrous connective tissue including basophilic globules of calcified lesion along with osteoid tissue circled by stratified squamous epithelium. Its solid fibrous connective tissue is made up of abundant spindle-shaped fibroblasts that cause a storiform pattern. COF contains woven bone and dispersed lamellar bone. Calcified woven bone separated as thin trabeculaes and edged by osteoblasts shows retiform pattern. The connective tissue contains round to ovoid calcified spherules appearing as psammoma bodies called 'cementum'. Pathology is also helpful to differentiate COF from fibrous dysplasia of which management is different. COF doesn't display any atypia, mitosis or malignancy and it is important to distinguish from fibrous dysplasia (FD). Additionally while COF contains both woven and lamellar bone, FD includes only woven bone not lamellar bone. 

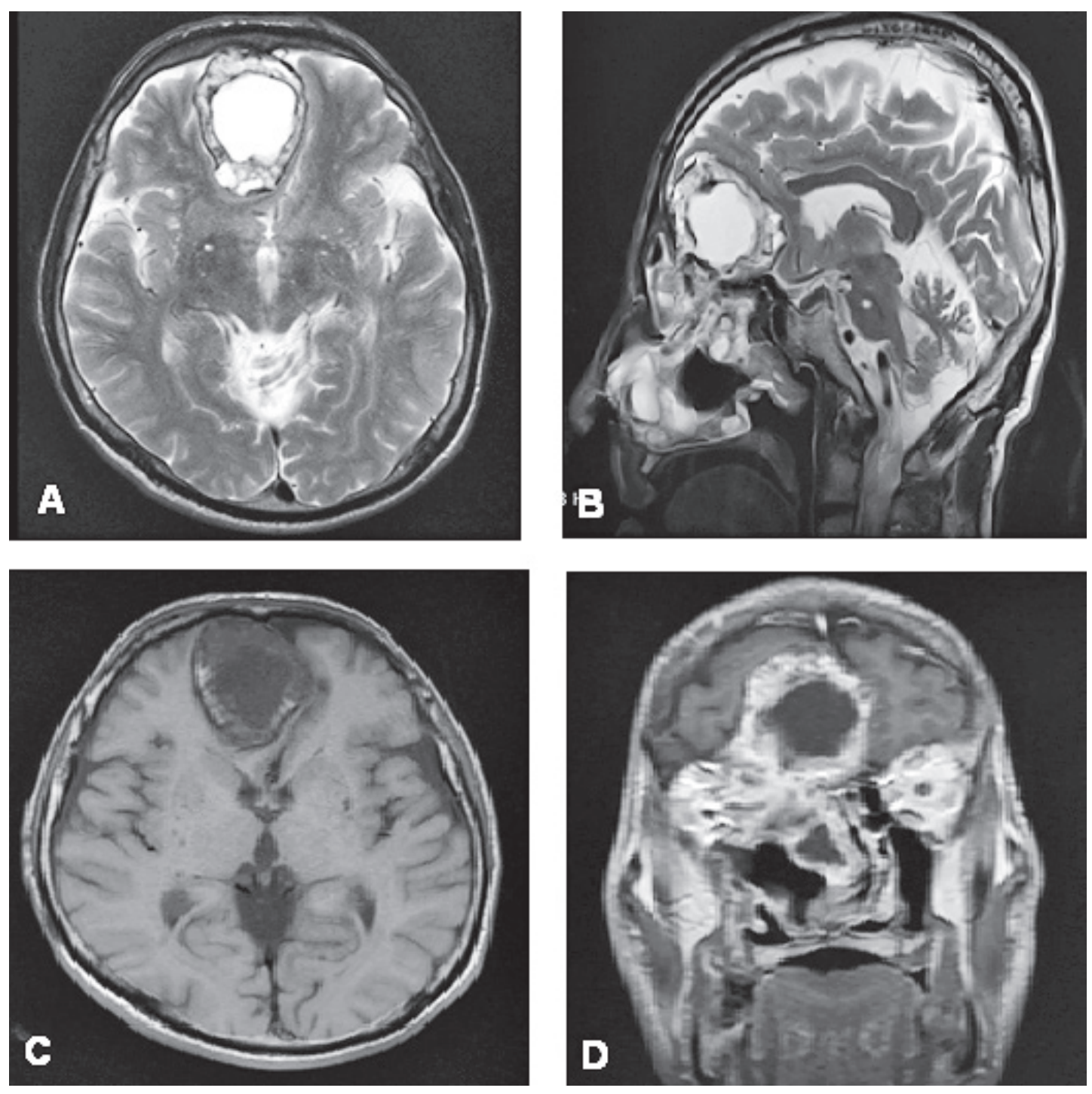

Fig. 3: T2W axial (A), sagittal (B), T1 W axial (C) and T1 W coronal postcontrast MRI images of the brain extra-axial mass in the frontal interhemispheric fissure (D). Mass is heterogenous with central T1 hypointense and T2 hyperintense area and peripheral heterogenous component with avid (but hetrerogenous) enhancement. There is no evidence of parenchymal invasion.

In some BFODs, histopathology doesn't manage to differentiate diagnosis. So radiography is important to help identify COF. A well-limited, smooth, corticated and sclerotic bordered lesion is characterized radiographically. There are three periods: Early, mixed and late period. While in the early period mainly radiolucent and cystic mass is seen, in the later period it gets central radiopaque bulk edged by radiolucent border, therefore similar to osteoblastoma.

Computer tomography shows a lytic zone circled by an eggshell-thin rim of bone. COF has a distinct border from adjacent tissue, however FD can't be separated from adjacent tissue clearly. CT also gives important information about mass extension and its relationship with neighbouring structures.

Magnetic resonance imaging gives low to intermediate signal intensity on T1-weighted images and changeable signal intensity on T2-weighted images. Ossified areas have low signal intensity, whereas central non-ossified lesions, cysts and included mucoceles have high signal intensity on T2-weighted images. Contrast agent increases the thick outer layer of the masses' signal densely. MRI is helpful to differentiate COF from FD. While FD always has hypointense signal intensity, COF may have hyperintense or hypointense signal intensity on T2-weighted image. With contrast agent in FD only the enlarged diploe gets 
signal intensity whereas in ossifying fibroma the outer shell and septa get signal enhancement.

Treatment is based on surgical excision or close follow-up. There is no curative medical treatment and radiotherapy isn't recommended. Surgical treatment strategy is so variable that either observation, local resection or total enucleating can be chosen according to the lesion's localization and size. Recurrence rate after surgical removal is variable. COF may be monitored with close control temporarily, if the patient who has no complications refuses surgery (10).

\section{References}

1. Eller R, Sillers M. Common fibro-osseous lesions of the paranasal sinuses. Otolaryngol Clin North Am 2006 Jun; 39(3): 585-600.
2. Lund VJ, Stammberger H, Nicolai P, et al. European position paper on endoscopic management of tumours of the nose, paranasal sinuses and skull base. Rhinol Suppl 2010; 22: 1-143

3. Barnes L, Eveson JW, Reichart P, Sidransky D. Pathology and genetics of head and neck tumours. In: World Health Organization classification of tumours. Lyon IARC, 2005: 319-20.

4. Eversole LR, Leider AS, Nelson K. Ossifying fibroma: a clinicopathologic study of sixty-four cases. Oral Surg Oral Med Oral Pathol. 1985; 60(5): 505-11.

5. Kansal R, Sharma A, Gaikwad N, Mahore A, Atul Goel A. Cemento-Ossifying Fibroma Presenting as a Posterior Fossa Mass Lesion Turkish Neurosurgery 2010; 20(2): 265-8.

6. Azaz B, Shteyer A, Soskolne WA. Fibro-osseous lesions of the jaws: a report of two uncommon cases. J Oral Med 1976; 31: 19-23.

7. Dominguete PR, Meyer TN, Alves FA, et al. Juvenile ossifying fibroma of the jaw. Br J Oral Maxillofac Surg 2008; 46: 480-1.

8. Eversole LR, Rovin S. Reactive lesions of the gingiva. J Oral Pathol 1972; 1:30-8. 9. Margo CE, Ragsdale BD, Perman KI, Zimmerman LE, Sweet DE. Psammomatoid (juvenile) ossifying fibroma of the orbit. Ophthalmology. 1985; 92(1): $150-9$.

10. Ledderose GJ, Stelter K, Becker S, Leunig A. Paranasal ossifying fibroma: endoscopic resection or wait and scan? Eur Arch Otorhinolaryngol 2011; 268: 999-1004.

Received: 13/06/2012

Accepted in revised form: 06/08/2012

\section{Corresponding author:}

Dr. Ibrahim Erdim, Bakırköy Dr. Sadi Konuk Eğitim ve Araştırma Hastanesi, Zuhuratbaba Mah. Tevfik Sağlam Cad. No:11, Bakırköy, Istanbul, Türkiye; e-mail: ibrahim_erdim@hotmail.com 